\title{
Meissner effect in a system of coupled one-dimensional superconducting wires: Monte Carlo simulations
}

\author{
Chunyin Qiu, ${ }^{1}$ Tiezheng Qian, ${ }^{1}$ and Ping Sheng ${ }^{2}$ \\ ${ }^{1}$ Department of Mathematics, The Hong Kong University of Science and Technology, Clear Water Bay, Kowloon, Hong Kong \\ ${ }^{2}$ Department of Physics and Institute of Nano Science and Technology, The Hong Kong University of Science and Technology, \\ Clear Water Bay, Kowloon, Hong Kong
}

(Received 27 August 2006; revised manuscript received 6 November 2006; published 11 January 2007)

\begin{abstract}
Motivated by the observation of superconducting behavior in $4 \AA$ carbon nanotube-zeolite composites, we use Monte Carlo simulations to evaluate the Meissner effect in a system of coupled one-dimensional superconducting wires. We model the system by using an anisotropic Ginzburg-Landau free energy functional, with distinct transverse and longitudinal coupling constants. By varying the degree of anisotropy, we obtain behaviors ranging from independent wires with suppressed superconducting transition, to an isotropic superconducting system. We numerically evaluate the Meissner effect for finite-sized superconducting domains. Comparison with the experimental data for carbon nanotube-zeolite composite shows the size of the phase-coherent, superconducting domains to be small, i.e., on the order of one or a few transverse coherence lengths. We provide a consistent estimate of the material parameters for the nanotube-zeolite composite, ranging from the penetration length of transverse magnetic field to the longitudinal and transverse coherence lengths.
\end{abstract}

DOI: 10.1103/PhysRevB.75.024504

PACS number(s): 74.20.De, 74.50.+r, 74.10.+v, 71.20.Tx

\section{INTRODUCTION}

Superconductivity in carbon nanotubes represents a competition between electron-phonon coupling strength, which favors small-diameters, ${ }^{1}$ and fluctuation effects, ${ }^{2}$ which become more and more dominant as the nanotube diameter decreases. In the limit of very small diameter carbon nanotubes, such as the $4 \AA$ nanotubes fabricated using the zeolite template, ${ }^{3}$ it could very well be that the fluctuation effects would completely suppress the superconducting transition. ${ }^{4}$ The carbon nanotube-zeolite composite is therefore a most interesting system, since when the $4 \AA$ carbon nanotubes are embedded in the ordered one-dimensional (1D) pores of the Aluminophosphate-five (AFI) zeolite, the wall-wall separation is $9.6 \AA$, small enough for Josephson coupling between the nanotubes to occur. This composite therefore represents a new paradigm for superconducting systems. In contrast to the high $T_{c}$ superconductors where the basic superconducting element is the two-dimensional (2D) $\mathrm{CuO}$ plane, here the basic element is the 1D carbon nanotube, ordered into twodimensional hexagonal arrays through the molecular structure of AFI zeolite, with precise and regular separations between the nanotubes. Indeed, superconducting behavior was observed in this composite, in which the Meissner effect indicates a rather high $T_{c} \sim 10$ to $15 \mathrm{~K}$. However, the fluctuation effects were also evident, as no sharp transition was observed. ${ }^{4}$

In this work, we use Monte Carlo simulation ${ }^{5}$ to directly evaluate the Meissner effect in a system of 1D superconducting wires, Josephson coupled to form a finite, anisotropic superconducting system,,${ }^{6,7}$ modeled by an anisotropic threedimensional (3D) Ginzburg-Landau (GL) free energy functional. Our numerical evaluation of the Meissner effect is accompanied by a comparison with the experimental data for carbon nanotube-zeolite composites, showing the size of the phase coherent, superconducting domains to be small, i.e., on the order of one or a few transverse coherence lengths. Based on the available experimental data and numerical simulation results, a consistent estimate of the penetration length of transverse magnetic field, plus the longitudinal and transverse coherence lengths, is obtained. In what follows, the main results are stated in Sec. II, followed by the formulation of the model and the relevant approach for numerical computation in Sec. III. Derivation of the formulas for the magnetic moment and magnetic susceptibility and their numerical evaluation are presented in Sec. IV, and the verification of the numerical calculation against known results is shown in Sec. V. In Sec. VI we present two relevant order parameters and their variations with temperature and model parameter values. By using our model, in Sec. VII we estimate the coherence lengths and magnetic penetration length of the nanotube-zeolite composite system, and compare the predictions of the model with experimental data of Ref. 4. In Sec. VIII the paper concludes with a few observations. Due to the intent of being self-contained, this paper has all the model details. But for those readers interested only in the physical implications of the model, Secs. II and VII are designed to be rather complete in describing the theory-experiment comparisons.

\section{STATEMENT OF MAIN RESULTS}

Compared to the numerical simulation in Ref. 4 which is essentially $1 \mathrm{D}$, the present work is based on an anisotropic 3D GL model, in which Josephson coupling between the superconducting nanowires is explicitly taken into account. By varying the interwire coupling strength,we obtain behaviors ranging from independent nanowires with suppressed superconducting transition, to an isotropic superconducting system. Numerical evaluation of the gap parameter shows that there is not much change from the 1D (Ref. 4) to the 3D model because the gap parameter measures the magnitude of local condensation, which is only weakly affected by the interwire coupling. But the present 3D simulation shows the 
diamagnetic response to be greatly enhanced by the interwire coupling that stabilizes the global phase coherence and maximizes the coherent diamagnetic response of the persistent current. That the diamagnetic response is size dependent in small systems indicates that the magnetic-field-induced suppression of superconductivity is also size dependent in these systems. Based on these numerical results for small 3D systems, the experimental data for the suppression of diamagnetic susceptibility ${ }^{4}$ can be used for estimating various material parameters, from the characteristic size of the superconducting domain (on the order of $100 \AA$ ) to the longitudinal and transverse coherence lengths (on the order of $1000 \AA$ and $100 \AA$ A,respectively), supplemented with an estimate of the penetration length of the transverse magnetic field (on the order of 1 micron) and a Wentzel-KramersBrillouin (WKB) tunneling probability between the wires $(\approx 1 / 100$, for the ratio between the longitudinal and transverse masses). The above estimates of the material parameters are consistent because they yield the right order of magnitude for the quenching (depairing) field ( $\sim 2$ Tesla) as observed experimentally. ${ }^{4} \mathrm{We}$ emphasize that the parameter estimation is made possible in the present anisotropic 3D GL model. The small domain size compared to the penetration length indicates that the absolute value of the diamagnetic susceptibility must be very small $\left(\sim 10^{-4} / 4 \pi\right)$, and hence the diamagnetic moment has to be measured in the high-field regime, approaching the quenching field. This indicates that the experimental data for diamagnetic susceptibility should be compared with the numerical results obtained for high fields. While qualitative agreement is seen in such a comparison, there is also a visible discrepancy that the numerically obtained temperature variation of the diamagnetic susceptibility is rather linear whereas experimentally the variation is much more nonlinear. ${ }^{4}$ This is attributed to the GL model itself in which a linear temperature dependence is assumed down to zero temperature, although such linear dependence usually works only in the vicinity of the transition temperature $T_{c}$.

\section{THE ANISOTROPIC GL FREE ENERGY AND ITS ISOTROPIZATION AND DISCRETIZATION}

The GL theory, based on a macroscopic wave function $\psi$, provides a simple but versatile description of superconductivity. ${ }^{8}$ As a generalization of the London theory, ${ }^{9}$ the GL theory is able to treat problems involving spatial variations and nonlinear responses to (strong) fields. Below we outline the computational GL model for the anisotropic system of coupled 1D superconducting wires.

The 3D anisotropic GL free energy functional can be expressed as

$$
\begin{aligned}
F_{G L}[\psi(\vec{r})]= & \int d^{3} r\left[\alpha|\psi|^{2}+\frac{\beta}{2}|\psi|^{4}\right. \\
& \left.+\sum_{\mu} \frac{1}{2 m_{\mu}}\left|\left(\frac{\hbar}{i} \partial_{\mu}-\frac{e^{*}}{c} A_{\mu}\right) \psi\right|^{2}\right] .
\end{aligned}
$$

Here $\psi=\psi(\vec{r})$ is a complex, spatially varying order param- eter, $e^{*}=2 e, \alpha=a_{o}\left(T-T_{c}^{o}\right), \beta=b_{o}$, with $a_{o}$ and $b_{o}$ being the two phenomenological parameters and $T_{c}^{o}$ the nominal phasetransition temperature, $m_{\mu}$ is the anisotropic effective mass in the $\mu$ direction with $\mu=x, y, z$, and $A_{\mu}$ is the $\mu$ component of the vector potential. The mean-field solution for $\psi(\vec{r})$ can be determined by directly minimizing the GL free energy functional when thermodynamic fluctuations are negligible. In order to include the fluctuation effects, one must evaluate various physical quantities from the free energy $F=-k_{B} T \ln Z$, where $k_{B}$ is the Boltzmann constant, $T$ is the temperature, and $Z$ is the partition function in the canonical ensemble: $Z=\int D \psi(\vec{r}) \exp \left\{-F_{G L}[\psi(\vec{r})] / k_{B} T\right\}$, in which $\int D \psi(\vec{r})$ denotes the functional integral. ${ }^{2}$ That is, the expectation value of a physical quantity $Q$ is to be evaluated as the ensemble average

$$
\langle Q\rangle=Z^{-1} \int D \psi(\vec{r}) Q \exp \left\{-F_{G L}[\psi(\vec{r})] / k_{B} T\right\} .
$$

Here and below \langle\rangle is used to denote the thermodynamic average in the canonical ensemble.

Given the anisotropic nature of the GL free energy, we adopt a scaling approach which rescales the anisotropic problem to an isotropic one, so long as the continuum GL description is applicable. This scaling approach has been widely used for quasi-2D high- $T_{c}$ superconductors. ${ }^{10}$ Essential to this approach is the isotropization of the gaugeinvariant gradient term in $F_{G L}$, which is realized by introducing the scaled coordinates $\bar{r}_{\mu}=r_{\mu} / \xi_{\mu 0}$, scaled vector potential $\bar{A}_{\mu}=A_{\mu} \xi_{\mu 0} / \Phi_{o}$, and scaled magnetic field $\bar{B}_{\mu}=B_{\mu} \delta_{0} / \xi_{\mu 0} \Phi_{o}$, where $\xi_{\mu 0}=\hbar / \sqrt{2 m_{\mu} a_{o} T_{c}^{o}}$ is the zero-temperature correlation length in the $\mu$ direction, $\delta_{0}=\xi_{x 0} \xi_{y 0} \xi_{z 0}$ is the corresponding volume unit, and $\Phi_{o}=h c / e^{*}$ is the flux quantum. Note that the scaled quantities introduced above are already dimensionless. Further introducing the dimensionless temperature $\bar{T}=T / T_{c}^{o}$ and the dimensionless order parameter $\bar{\psi}$ through $|\bar{\psi}|^{2}=|\psi|^{2} /\left|\psi_{0}\right|^{2}$, with $\left|\psi_{0}\right|^{2}=a_{o} T_{c}^{o} / b_{o}$ being the zerotemperature mean-field value of $|\psi|^{2}$ (the number density of superconducting Cooper pairs), we obtain the GL free energy functional in the dimensionless form

$$
\begin{aligned}
\bar{F}_{G L}(\bar{\psi})= & \int d^{3} \bar{r}\left[2(\bar{T}-1)|\bar{\psi}|^{2}+|\bar{\psi}|^{4}\right. \\
& \left.+\sum_{\mu=1}^{3} 2\left|\left(-i \bar{\partial}_{\mu}-2 \pi \bar{A}_{\mu}\right) \bar{\psi}\right|^{2}\right],
\end{aligned}
$$

defined by $\bar{F}_{G L}=F_{G L} / \varepsilon_{o} k_{B} T_{c}^{o}$, with $\varepsilon_{0}=a_{o}^{2} T_{c}^{o 2} \delta_{0} / 2 b_{o} k_{B} T_{c}^{o}$ being the zero-temperature condensation energy in units of $k_{B} T_{c}^{o}$, for the characteristic volume $\delta_{0}=\xi_{x 0} \xi_{y 0} \xi_{z 0}$. Below we assume that the wires are aligned along the $z$ direction, and the transverse coherence lengths $\xi_{x 0}$ and $\xi_{y 0}$ are smaller than the longitudinal coherence length $\xi_{z 0}$ because of weak interwire coupling.

Since it is our intent to compare our simulation results with the data from the nanotube-zeolite composites, it would be interesting to estimate the relative ratio of electronic bandwidths for the longitudinal and transverse couplings, in- 
volving an evaluation of the WKB tunneling probability (coupling strength) between the wires (i.e., the nanotubes). As the zeolite's bandgap is $\sim 5.7 \mathrm{eV}$ (Ref. 11) and if the chemical potential is located at the middle of the gap, then the tunneling barrier height is $U \sim 3 \mathrm{eV}$. With a nanotube-tonanotube wall-to-wall separation of $l=9.6 \AA$, the tunneling probability is $\exp \left(-\sqrt{2 m_{z} U} l / \hbar\right) \sim 1 / 100$ for an effective tunneling mass (along the longitudinal nanotube axis) of $m_{z}=0.24 m_{e}$ [from the LDA calculations for the $(5,0)$ carbon nanotubes ${ }^{12}$, i.e., the transverse coupling is reasonably strong in the nanotube-zeolite composite. This ratio should be comparable to that between the longitudinal and transverse effective masses. Since the coherence length is proportional to the inverse square root of the effective mass, the above estimate implies that the transverse coherence length is $\sim 0.1$ times the longitudinal coherence length, i.e., $\xi_{x 0} \approx \xi_{y 0} \approx 0.1 \xi_{z 0}$. This would serve as a guide to our parameter estimation in Sec. VII.

To numerically evaluate the functional integrals by Monte Carlo simulation, ${ }^{5}$ we employ the following discretization scheme. Consider a finite sample in the computational domain whose spatial extensions in the three directions are given by $\bar{L}_{\mu}=L_{\mu} / \xi_{\mu 0}$ for $\mu=x, y, z$, respectively. In each direction $\bar{L}_{\mu}$ is discretized by using the dimensionless spacing $\bar{d}$. That is, the length $L_{\mu}$ in real space is discretized by the spacing unit $d_{\mu}=\bar{d} \xi_{\mu 0}$, i.e., different spacing units are used in spatial directions with different correlation lengths. The same dimensionless spacing $\bar{d}$ for discretization in all the directions leads to a simple cubic lattice in the computational domain, which facilitates faster numerical computation. This is one of the many advantages that arise from the isotropization procedure. In terms of the discrete grid coordinates $(i, j, k)$, the dimensionless spatial coordinates $\bar{x}, \bar{y}$, and $\bar{z}$ can be expressed as $\bar{x}(i)=[i-(m+1) / 2] \bar{d}, \bar{y}(j)=[j-(n+1) / 2] \bar{d}$, and $\bar{z}(k)=[k-(l+1) / 2] \bar{d}$, where $m, n, l$ are the grid numbers in the $x, y, z$ directions. So the total grid number is $N_{G}=m n l$ and the sample size is $\bar{L}_{x} \times \bar{L}_{y} \times \bar{L}_{z}=N_{G} \bar{d}^{3}$ in the computational domain. The discretized dimensionless GL free energy functional is given by

$$
\begin{aligned}
F_{G L}(\{\bar{\psi}(i, j, k)\})= & \bar{d}^{3} \sum_{i, j, k}\left[2(\bar{T}-1)|\bar{\psi}(i, j, k)|^{2}+|\bar{\psi}(i, j, k)|^{4}\right] \\
& +2 \bar{d}\left\{\sum_{i>1, j, k} \mid e^{-i \Delta \varphi_{x}(i, j, k)} \bar{\psi}(i, j, k)\right. \\
& -\left.\bar{\psi}(i-1, j, k)\right|^{2}+\sum_{i, j>1, k} \mid e^{-i \Delta \varphi_{y}(i, j, k)} \bar{\psi}(i, j, k) \\
& -\left.\bar{\psi}(i, j-1, k)\right|^{2}+\sum_{i, j, k>1} \mid e^{-i \Delta \varphi_{z}(i, j, k)} \bar{\psi}(i, j, k) \\
& -\bar{\psi}(i, j, k-1) \mid\}
\end{aligned}
$$

where $\Delta \varphi_{\mu}(i, j, k)=2 \pi \bar{A}_{\mu}(i, j, k) \bar{d}$ is the phase change due to the gauge field. Based on the above free energy functional, the partition function is of the form

$$
Z=\int \prod_{\ell=1}^{N_{G}} d \bar{\psi}_{\ell} \exp \left[-\varepsilon_{o} \bar{F}_{G L}\left(\left\{\bar{\psi}_{\ell}\right\}\right) / \bar{T}\right]
$$

with the subscript $\ell$ standing for $(i, j, k)$. The ensemble average may be expressed as

$$
\langle\bar{Q}\rangle=Z^{-1} \int \prod_{\ell=1}^{N_{G}} d \bar{\psi}_{\ell} \bar{Q} \exp \left[-\varepsilon_{o} \bar{F}_{G L}\left(\left\{\bar{\psi}_{\ell}\right\}\right) / \bar{T}\right],
$$

where $\bar{Q}=Q / \hat{Q}$, with $\hat{Q}$ representing some (natural) unit for $Q$. Based on the above expressions for $Z$ and $\langle\bar{Q}\rangle$, various expectation values in the canonical ensemble can be evaluated by using the Metropolis importance sampling algorithm. ${ }^{5}$

\section{MAGNETIC MOMENT AND SUSCEPTIBILITY}

The main purpose of this paper is to investigate the diamagnetic response of the coupled 1D superconducting wires. For simplicity, we assume that the transverse external field is applied along the $x$ direction, i.e., $\vec{H}=(H, 0,0)$. The equilibrium properties of the system, the diamagnetic moment (along $x$ ) and its corresponding susceptibility, can be expressed as

$$
\langle m[\psi(\vec{r})]\rangle=-\frac{\partial F}{\partial B}=-\left\langle\frac{\partial}{\partial B} F_{G L}[\psi(\vec{r})]\right\rangle,
$$

and

$$
\begin{aligned}
\langle\chi[\psi(\vec{r})]\rangle= & -\frac{1}{V} \frac{\partial^{2} F}{\partial B^{2}} \\
= & -\frac{1}{V}\left\{\left\langle\frac{\partial^{2} F_{G L}}{\partial B^{2}}\right\rangle\right. \\
& \left.-\frac{1}{k_{B} T}\left[\left\langle\left(\frac{\partial F_{G L}}{\partial B}\right)^{2}\right\rangle-\left(\left\langle\frac{\partial F_{G L}}{\partial B}\right\rangle\right)^{2}\right]\right\},
\end{aligned}
$$

with $V$ the total volume. In principle, $\vec{B}$ refers to the local magnetic field, which has already taken into account the contribution from the diamagnetic persistent current and thus needs to be solved self-consistently. However, for the samples to be considered here, the diamagnetic response is actually very weak (as indicated by the magnitude of the diamagnetic susceptibility being on the order of $1 \%$ or less). Therefore we may simply adopt $\vec{H}=(H, 0,0)$ and $\vec{B} \cong \vec{H}$ for the purpose of computing $\langle m\rangle$ and $\langle\chi\rangle$ to the leading order. ${ }^{13}$ We choose the symmetric gauge $\vec{A} \cong \vec{B} \times \vec{r} / 2$. As expected, this gauge can greatly speed up the Monte Carlo simulations compared to other choices. The dimensionless field strength is $\bar{B}=B \delta_{0} / \xi_{x 0} \Phi_{o}$ and the dimensionless vector potential is distributed according to $\bar{A}_{x}(i, j, k)=0, \bar{A}_{y}(i, j, k)=-\bar{B} \bar{z}(k) / 2$, and $\bar{A}_{z}(i, j, k)=\bar{B} \bar{y}(j) / 2$. By scaling the diamagnetic moment $\langle m\rangle$ with $\hat{m}=\varepsilon_{o} k_{B} T_{c}^{o} \delta_{0} / \xi_{x 0} \Phi_{o}$, we obtain the dimensionless moment in the form of 


$$
\left\langle\bar{m}\left(\left\{\bar{\psi}_{\ell}\right\}\right)\right\rangle=-4 \pi \bar{d}^{2}\left\langle\operatorname{Im}\left[\sum_{i, j>1, k} e^{-i \Delta \varphi_{y}(i, j, k)} \bar{\psi}(i, j, k) \bar{\psi}^{*}(i, j-1, k) \bar{z}(k)-\sum_{i-1, j, k} e^{-i \Delta \varphi_{z}(i, j, k)} \bar{\psi}(i, j, k) \bar{\psi} *(i, j, k-1) \bar{y}(j)\right]\right\rangle,
$$

for numerical computation. By scaling the susceptibility $\langle\chi\rangle$ by $\hat{\chi}=\varepsilon_{o} k_{B} T_{c}^{o} \delta_{0} /\left(\xi_{x 0} \Phi_{o}\right)^{2}$, we obtain the dimensionless susceptibility in the form of

$$
\left\langle\bar{\chi}\left(\left\{\bar{\psi}_{\ell}\right\}\right)\right\rangle=\left\langle\bar{\chi}_{1}\left(\left\{\bar{\psi}_{\ell}\right\}\right)\right\rangle+\left\langle\bar{\chi}_{2}\left(\left\{\bar{\psi}_{\ell}\right\}\right)\right\rangle+\left\langle\bar{\chi}_{3}\left(\left\{\bar{\psi}_{\ell}\right\}\right)\right\rangle,
$$

where

$$
\left\langle\bar{\chi}_{1}\left(\left\{\bar{\psi}_{\ell}\right\}\right)\right\rangle=-4 \pi^{2} \bar{d}^{3}\left\langle\operatorname{Re}\left[\sum_{i, j>1, k} e^{-i \Delta \varphi_{y}(i, j, k)} \bar{\psi}(i, j, k) \bar{\psi}^{*}(i, j-1, k) \bar{z}^{2}(k)+\sum_{i=1, j, k} e^{-i \Delta \varphi_{z}(i, j, k)} \bar{\psi}(i, j, k) \bar{\psi}^{*}(i, j, k-1) \bar{y}^{2}(j)\right]\right\rangle / \bar{V},
$$

$$
\begin{gathered}
\left\langle\bar{\chi}_{2}\left(\left\{\bar{\psi}_{\ell}\right\}\right)\right\rangle=\left\langle\left[\bar{m}\left(\left\{\bar{\psi}_{\ell}\right\}\right)\right]^{2}\right\rangle \varepsilon_{o} / \bar{T} \bar{V}, \\
\left\langle\bar{\chi}_{3}\left(\left\{\bar{\psi}_{\ell}\right\}\right)\right\rangle=-\left[\left\langle\bar{m}\left(\left\{\bar{\psi}_{\ell}\right\}\right)\right\rangle\right]^{2} \varepsilon_{o} / \bar{T} \bar{V},
\end{gathered}
$$

with $\bar{V}=V / \delta_{0}$ being the dimensionless volume. Here "*”" denotes the complex conjugate, and "Im" and "Re" mean the imaginary and real parts of the quantity that follows. It is to be noted that the units $\hat{m}$ and $\hat{\chi}$ for $\langle\bar{m}\rangle$ and $\langle\bar{\chi}\rangle$ contain the information on how $\langle m\rangle$ and $\langle\chi\rangle$ are influenced by the anisotropy, measured by the ratio between the three zerotemperature correlation lengths $\xi_{\mu 0}$.

To compare with the experimental data obtained for nonzero external fields, we have also calculated the diamagnetic susceptibility from

$$
\langle\chi[\psi(\vec{r})]\rangle=\frac{\langle m[\psi(\vec{r})]\rangle}{V B},
$$

using the corresponding dimensionless expression

$$
\left\langle\bar{\chi}\left(\left\{\bar{\psi}_{\ell}\right\}\right)\right\rangle=\frac{\left\langle\bar{m}\left(\left\{\bar{\psi}_{\ell}\right\}\right)\right\rangle}{\overline{V B}} .
$$

We note that the two definitions for $\langle\chi\rangle$ agree with each other in the limit of $B \rightarrow 0$ because for small applied fields $\langle m\rangle$ is linear in $B$.

Based on the isotropization described in Sec. III, the anisotropic character in the diamagnetic response has been absorbed into the units $\hat{m}$ and $\hat{\chi}$. Consequently, the numerical evaluation of $\langle\bar{m}\rangle$ and $\langle\bar{\chi}\rangle$ is to be carried out for an isotropic system controlled by only two dimensionless parameters: (i) the intrinsic parameter $\varepsilon_{0}$ which measures both the condensation energy for each "grain" and the coupling energy between the nearest-neighbored grains, and (ii) the geometric shape and size of the system measured in the space of $\bar{r}_{\mu}$ $=r_{\mu} / \xi_{\mu 0}$.

\section{MODEL AND NUMERICAL VERIFICATIONS}

To model our experimental samples, it is noted that the superconducting nanotube-zeolite composite is most likely type II in character, since we expect the transverse coherence length between the nanotubes to be smaller than the penetration length of the transverse magnetic field (in the $x$-direction), i.e., $\xi_{x 0} \approx \xi_{y 0}<\lambda_{z 0}$, with $\lambda_{z 0}$ defined by $1 / \lambda_{z 0}^{2}$ $=4 \pi e^{* 2}\left|\psi_{0}\right|^{2} / m_{z} c^{2}$. (The diamagnetic supercurrent induced by the transverse field flows along the wires in the $z$-direction, and hence the effective mass $m_{z}$ is the one that is relevant.) However, in anticipation of the fact that the experimentally measured Meissner effect arises from superconducting regions that are only on the order of the transverse coherence length (see Sec. VII for parameter estimation), here we confine ourselves to modeling finite-sized samples that are of similar sizes. In these cases whether the superconducting character is type I or type II makes essentially no difference, since the magnetic field inhomogeneities in type II superconductors (such as the flux lattice) are manifest only at the scale of (at least) several penetration lengths. Below we first verify our numerical simulations against known results for thin samples with negligible magnetic field inhomogeneities, as evidenced by the small magnitude of the susceptibility. Note that the susceptibilities calculated here are in units of $\hat{\chi}=\varepsilon_{o} k_{B} T_{c}^{o} \delta_{0} /\left(\xi_{x 0} \Phi_{o}\right)^{2}=\xi_{y 0}^{2} / 64 \pi^{3} \lambda_{z 0}^{2} \ll 1 / 2000$.

We have carried out numerical simulations by using $\bar{d}$ $=1$, i.e., $d_{\mu}=\xi_{\mu 0}$ in discretizing the space. Such a discretization allows all the fluctuations of wavelengths $\geq \xi_{\mu 0}$ to occur. This naturally takes into account the dominant fluctuations near the critical temperature, which are of wavelengths $\gg \xi_{\mu 0}$. Physically, the order parameter $\psi(\vec{r})$ is measured at a discrete set of points that are further apart than $\xi_{\mu 0}$, since the GL theory is no longer valid for fluctuations on a scale shorter than $\xi_{\mu 0 \cdot}{ }^{14,15}$

The validity of our computational model was checked from three different perspectives. First, the gauge invariance of the physical observables $\langle\bar{m}\rangle$ and $\langle\bar{\chi}\rangle$ was verified by using gauges other than the symmetric one. Second, for small $\bar{B}$, the susceptibility $\langle\bar{\chi}\rangle$ was directly measured according to $\langle\bar{\chi}\rangle=\left\langle\bar{\chi}_{1}\right\rangle+\left\langle\bar{\chi}_{2}\right\rangle+\left\langle\bar{\chi}_{3}\right\rangle$, and good agreement was obtained in a comparison with $\langle\bar{\chi}\rangle=\langle\bar{m}\rangle / \overline{V B}$ evaluated from the measured $\langle\bar{m}\rangle$. Third, the low-temperature, small-field behaviors obtained for finite samples were compared with the theoretical predictions of the London equation, ${ }^{9}$ which assumes perfect phase coherence throughout the system, a condition indeed realized at low temperatures. For example, the London equa- 

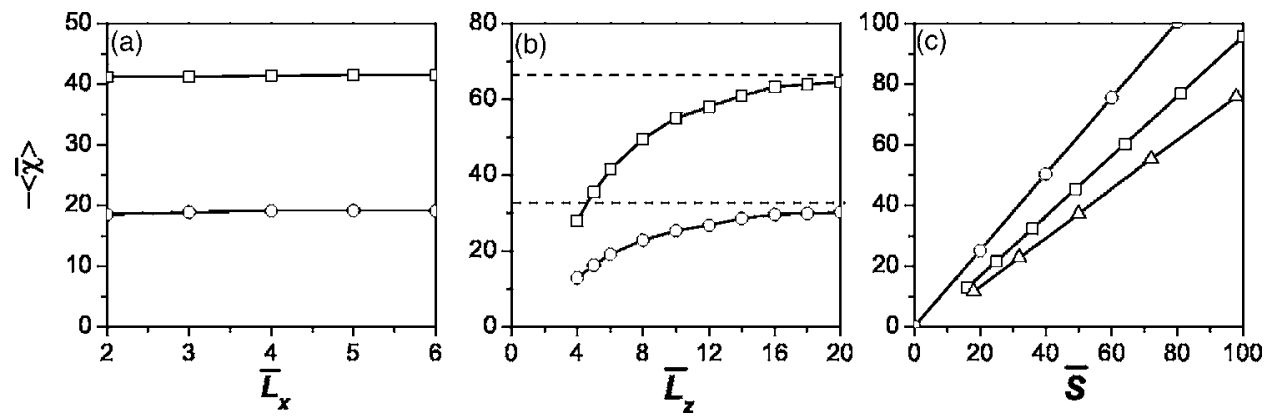

FIG. 1. (a) Zero-field diamagnetic susceptibility at temperatures $\bar{T}=0.6$ (squares) and 0.8 (circles), evaluated for different rectangular samples. Here the thickness $\bar{L}_{x}$ is varied while the cross-sectional area is fixed at $\bar{L}_{y} \times \bar{L}_{z}=4 \times 6$, with $\varepsilon_{0}=10$. It is seen that the susceptibility is independent of the thickness as predicted by the London equation. (b) Zero-field diamagnetic susceptibility at temperatures $\bar{T}=0.6$ (squares) and 0.8 (circles), evaluated for different rectangular samples. Here the length $\bar{L}_{z}$ is varied while the thickness $\bar{L}_{x}$ is fixed at 5 and the width $\bar{L}_{y}$ fixed at 4 , with $\varepsilon_{0}=10$. The dashed lines denote the values predicted by the London equation for narrow stripes (of effective widths 3.53 and 3.45), i.e., $\bar{\chi}=-4 \pi^{2} \bar{L}_{y}^{2}(1-\bar{T}) / 3$ for $\bar{L}_{z} / \bar{L}_{y} \rightarrow \infty$. (c) Zero-field diamagnetic susceptibility at temperature $\bar{T}=0.8$, evaluated for rectangular samples of different cross-sectional aspect ratios $\bar{L}_{z} / \bar{L}_{y}=1$ (squares) and 0.5 (triangles). Here the cross-sectional area $\bar{S}=\bar{L}_{y} \bar{L}_{z}$ is varied and the thickness $\bar{L}_{x}$ is fixed at 5 , with $\varepsilon_{0}=10$. The circles represent the results predicted by the London equation for a cylinder, with the applied field parallel to the cylindrical axis, i.e., $\bar{\chi} \approx-2 \pi(1-\bar{T}) \bar{S}$, where $\bar{S}$ is the dimensionless cross-sectional area. These results show that cross-sectional shape of higher symmetry leads to larger diamagnetic response.

tion predicts that for any sample with a uniform thickness in the field direction, the susceptibility is independent of that thickness. This has been confirmed in Fig. 1(a), obtained for a set of rectangular samples of the same cross section $\left(\bar{L}_{y}\right.$ and $\left.\bar{L}_{z}\right)$ but different thicknesses $\left(\bar{L}_{x}\right.$ in the field direction). Then, with the thickness $\bar{L}_{x}$ and width $\bar{L}_{y}$ both fixed and the length $\bar{L}_{z}$ increased from 4 to 20 , the susceptibility was observed to increase monotonically but more and more slowly, approaching some saturation value that can be predicted by solving the London equation for a narrow stripe. ${ }^{8}$ The results shown in Fig. 1(b) indicate that the shape of the sample's cross section can considerably affect the magnitude of the susceptibility. Based on the London equation, it is easy to prove that for samples of uniform thickness $\bar{L}_{x}$ and rectangular cross section $\bar{L}_{y} \times \bar{L}_{z}$, the susceptibility is of the scaling form $f\left(\bar{L}_{z} / \bar{L}_{y}\right) \bar{L}_{y}^{2}$, in which $f(x)$ is a function of the aspect ratio $\bar{L}_{z} / \bar{L}_{y}$, approaching saturation (for the case of stripe) as $\bar{L}_{z} / \bar{L}_{y} \rightarrow \infty$. This scaling has indeed been well verified as seen in Fig. 1(c).

\section{ORDER PARAMETERS AND LIMITING BEHAVIORS}

To investigate the effect of the energy parameter $\varepsilon_{0}$, we fix the sample size with $\bar{L}_{x}=\bar{L}_{y}=\bar{L}_{z}=20$ and evaluate two intrinsic properties of the system

$$
\eta \equiv N_{G}^{-1}\left\langle|| \sum_{\ell=1}^{N_{G}} \exp \left(i \phi_{\ell}\right) \mid\right\rangle
$$

and

$$
\gamma \equiv N_{G}^{-1}\left\langle\sum_{\ell=1}^{N_{G}}\left|\bar{\psi}_{\ell}\right|^{2}\right\rangle,
$$

where $\phi_{\ell}$ is the phase of the complex order parameter $\psi_{\ell}$. Here $\eta$ is the global phase-ordering parameter, measuring the overall phase coherence throughout the sample and $\gamma$ is the mean-square gap parameter. The temperature dependence of $\eta$ is plotted in Fig. 2 for $\varepsilon_{0}$ varied in a wide range. It shows that the system undergoes a phase-ordering transition at the critical temperature $T_{c}$, below which there is global phase coherence throughout the whole system, i.e., $\eta$ displays a jump from 0 to 1 as $T_{c}$ is approached from above. In our Monte Carlo simulations, the jump of $\eta$ is rounded because of the finite size of the samples. Figure 2 also shows that in the regime of weak coupling (small $\varepsilon_{0}$ ), the phase-ordering transition temperature $T_{c}$ can be appreciably lower than the nominal transition temperature $T_{c}^{o}$. This suppression of transition temperature indicates that when the interwire coupling is sufficiently weak ( $\xi_{x 0} \approx \xi_{y 0} \rightarrow 0$ compared to $\xi_{z 0}$ and thus $\left.\varepsilon_{0} \propto \xi_{x 0} \xi_{y 0} \rightarrow 0\right)$, the extreme anisotropy of the system makes

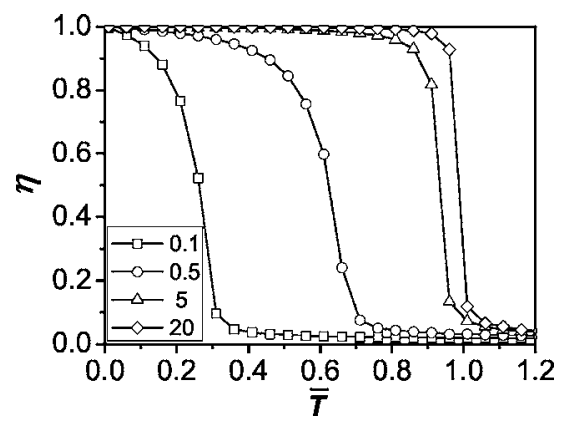

FIG. 2. Phase-ordering parameter $\eta$ as a function of the temperature, evaluated for different values of $\varepsilon_{0}$, with the dimensions of the sample fixed at $\bar{L}_{x}=\bar{L}_{y}=\bar{L}_{z}=20$. 


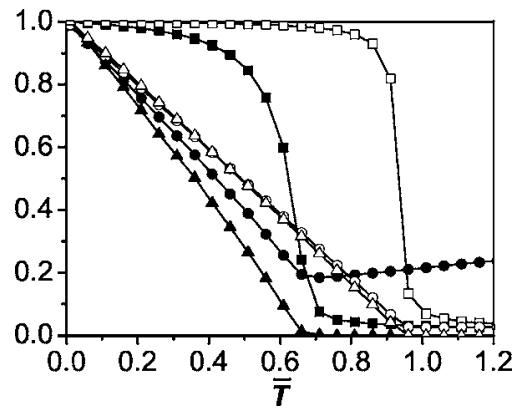

FIG. 3. Variations of $\eta$ (squares), $\gamma$ (circles), and the zero-field diamagnetic susceptibility $\langle\bar{\chi}\rangle$ (triangles) with the temperature, evaluated for $\varepsilon_{0}=0.5$ (solid symbols) and 5 (open symbols) with the dimensions of the sample fixed at $\bar{L}_{x}=\bar{L}_{y}=\bar{L}_{z}=20$. Here $-\langle\bar{\chi}\rangle / \chi_{0}>0$ is plotted for $\langle\bar{\chi}\rangle$, with $\chi_{0}$ the magnitude of $\langle\bar{\chi}\rangle$ at zero temperature.

$T_{c}$ approach zero, the transition temperature for independent 1D wires according to the Hohenberg-Mermin-Wagner theorem. ${ }^{16}$ On the other hand, when the coupling parameter $\varepsilon_{0}$ is large enough, the critical temperature $T_{c}$ approaches $T_{c}^{o}$. That the phase-ordering transition may be well separated from $T_{c}^{0}$ has been observed in the Monte Carlo simulation for a system of superconducting grains coupled together by Josephson coupling. ${ }^{17,18}$ In that system the grains are sufficiently large. This leads to the single-grain transition of $\gamma$ at the mean-field transition point $T_{c}^{o}$, though the global phaseordering transition may occur at a temperature below $T_{c}^{o}$ for weak Josephson coupling. In the present system modeled by $F_{G L}=\varepsilon_{o} k_{B} T_{c}^{o} \bar{F}_{G L}$, the parameter $\varepsilon_{0}$ measures both the singlegrain energy and the intergrain coupling. When the intergrain coupling is so weak that $T_{c}$ is well separated from $T_{c}^{o}$, the single-grain energy scale is already small enough to make the temperature dependence of $\gamma$ appreciably different from the mean-field behavior.

The variation of $\gamma$ is well approximated by the mean-field relation $\gamma=1-\bar{T}$ for $\bar{T}<1$ when $\varepsilon_{0}$ is sufficiently large and $T_{c}$ is very close to $T_{c}^{o}$, i.e., $\bar{T}_{c} \equiv T_{c} / T_{c}^{o} \cong 1$. For small $\varepsilon_{0}$ with $\bar{T}_{c}$ appreciably below 1 , the variation of $\gamma$ displays two different regimes separated by $\bar{T}_{c}$, exhibiting a nonmonotonic temperature dependence. As shown in Fig. 3, below $\bar{T}_{c}$ the dependence of $\gamma$ on $\bar{T}$ is approximately linear (especially for $\bar{T} \rightarrow 0$ ), with $\gamma$ decreasing with the increasing $\bar{T}$. This "lowtemperature" behavior can be approximately described by $\gamma=1-\bar{T}-c \bar{T} / \varepsilon_{0}$ where $1-\bar{T}$ comes from the mean-field contribution and $-c \bar{T} / \varepsilon_{0}$ is the correction due to thermal fluctuations. Note that $\bar{T} / \varepsilon_{0}$ measures the ratio of the thermal energy $k_{B} T$ to the intrinsic energy scale $\varepsilon_{o} k_{B} T_{c}^{o}$, and naturally the leading-order correction is linear in $\bar{T} / \varepsilon_{0}$. Above $\bar{T}_{c}$ the variation of $\gamma$ is reversed: $\gamma$ increases with the increasing temperature. In this regime, there is no long-range phase coherence; only one or a few grains are fluctuating coherently. As a consequence, the magnitude of $\gamma$ is dominated by the incoherent fluctuations, which increases the value of $\gamma$ as temperature increases.

Physically the magnitude of the diamagnetic susceptibility is closely related to the range of phase coherence. ${ }^{9}$ There- fore, the behaviors of $\eta$ and $\langle\bar{\chi}\rangle$ must be closely related. Above the critical temperature $T_{c}$, the global phase coherence is lost and $\eta \rightarrow 0$, hence $\langle\bar{\chi}\rangle$ approaches zero as well (see Fig. 3). While the tail of $\eta$ is due to the finite-size effect, that of $\langle\bar{\chi}\rangle$ arises from the short-range phase coherence remaining in the fluctuating system. ${ }^{19}$ Now using Fig. 3 we show that below $T_{c}$, the temperature dependence of $\langle\bar{\chi}\rangle$ is jointly controlled by the two intrinsic parameters $\eta$ and $\gamma$. (Note that to study the intrinsic properties of the system, a fairly large cross-sectional area $\bar{L}_{y} \times \bar{L}_{z}=20 \times 20$ has been used in producing $\langle\bar{\chi}\rangle$ shown in Fig. 3. That the magnetic field inhomogeneities is still negligible here requires the sample to be in the extreme type II regime, i.e., $\lambda_{z 0} \gg \xi_{y 0}$ and the susceptibility unit $\hat{\chi}=\xi_{y 0}^{2} / 64 \pi^{3} \lambda_{z 0}^{2}$ is so small that $|\langle\bar{\chi}\rangle| \hat{\chi} \ll 1$ can still be realized.)

For a finite sample at temperatures well below $\bar{T}_{c}$, the global phase coherence is maintained, as evidenced by $\eta \approx 1$. Consequently, the London theory is applicable and it predicts that $\langle\bar{\chi}\rangle$ is proportional to the mean-square gap parameter $\gamma$. That means in the low temperature regime, the $\bar{T}$ dependence of $-\langle\bar{\chi}\rangle$ (or $-\langle\bar{\chi}\rangle / \chi_{0}$ with $\chi_{0}$ the magnitude of $\langle\bar{\chi}\rangle$ at zero temperature) is almost the same as that of $\gamma$, given by $\gamma=1-\bar{T}-c \bar{T} / \varepsilon_{0}$ approximately. As the temperature is raised towards $\bar{T}_{c}$, however, the $\bar{T}$ dependence of $-\langle\bar{\chi}\rangle$ has to gradually deviate from that of $\gamma$. This is because $\langle\bar{\chi}\rangle$ tends to vanish at $\bar{T}_{c}$, where the global phase coherence is lost, whereas $\gamma$, extrapolated from the linear dependence $\gamma=1-\bar{T}-c \bar{T} / \varepsilon_{0}$ for $\bar{T} \rightarrow 0$, remains well above zero at $\bar{T}_{c}$. Obviously, for sufficiently large $\varepsilon_{0}$, since $\eta$ and $\gamma$ tend to vanish at $\bar{T}_{c} \cong 1$ simultaneously, the temperature dependence of $-\langle\bar{\chi}\rangle$ (or $\left.-\langle\bar{\chi}\rangle / \chi_{0}\right)$ stays very close to that of $\gamma$, i.e., $-\langle\bar{\chi}\rangle / \chi_{0} \cong 1-\bar{T}$ for $\bar{T}<1$.

\section{PARAMETERS ESTIMATION AND COMPARISON WITH EXPERIMENT}

To make an appropriate parameter estimation for the actual samples, we note that according to the estimate of the transverse tunneling probability in Sec. III, the transverse coupling is reasonably strong between the carbon nanotubes, and the anisotropy of the system allows us to follow the general directions set by the conventional theory of superconductivity. For conventional bulk superconductors, only $\sim 10^{-4}$ of the electrons are involved in condensation, with the density of Cooper pairs roughly given by $10^{-4} n \sim 10^{18} / \mathrm{cm}^{3}$, where $n \sim 10^{22} / \mathrm{cm}^{3}$ is the density of conducting electrons. Accordingly, we start from $\left|\psi_{0}\right|^{2} \sim 10^{18} / \mathrm{cm}^{3}$ as the density of Cooper pairs at zero temperature. Together with the longitudinal effective mass of $m_{z}=0.24 m_{e}$, we estimate the penetration length of transverse magnetic field to be $\lambda_{z 0}$ $=\sqrt{m_{z} c^{2} / 4 \pi e^{* 2}\left|\psi_{0}\right|^{2}} \cong 1.3 \times 10^{-4} \mathrm{~cm}$. (The diamagnetic supercurrent induced by the transverse field flows along the wires in the $z$-direction, and hence the effective mass $m_{z}$ is the one that is relevant.)

To estimate the characteristic size of the phase-coherent superconducting domains in the nanotube-zeolite composite, 
we turn to the experimental data for diamagnetic susceptibility, which show that, at sufficiently low temperatures, the diamagnetism is completely suppressed when the applied transverse field is as high as $H_{c c}>2.0$ Tesla. This field strength is $\sim 100$ times higher than the typical critical field $H_{c}$, usually on the order of a few hundred Gauss (estimated from the condensation energy density $H_{c}^{2} / 8 \pi \sim 10^{-4} n k_{B} T_{c}^{o}$ ). Such a huge difference indicates that the phase-coherent domain is actually much smaller than the London penetration length $\lambda_{L}$. It is well known that for a superconducting stripe of width $D\left(\ll \lambda_{L}\right)$, its susceptibility is reduced by a factor of $D^{2} / \lambda_{L}^{2}$. As a consequence, to suppress the superconductivity of the stripe, the applied field $H_{c c}$ has to be larger than the bulk critical field $H_{c}$ by a factor of $\lambda_{L} / D$. In the present anisotropic case, if the characteristic width of the coherent domain in the cross section normal to the transverse field is $D$, then the critical field should be approximately amplified by a factor of $\lambda_{z 0} / D$. If we assume that at zero temperature, $\lambda_{z 0} / D \approx 100$ and $\lambda_{z 0} \approx 10^{-4} \mathrm{~cm}$ as estimated above, then $D \approx 10^{-6} \mathrm{~cm}$.

Due to the inevitable distribution of the superconducting domains in actual samples, the Meissner response of the system is argued to be dominated by those with transverse size on the order of a few transverse coherence lengths, i.e., $D \sim \xi_{y 0}$. The reason is that for those domains smaller than a transverse coherence length, which can be very numerous, the superconductivity is suppressed. While there could be domains with transverse dimensions significantly larger than a few transverse coherence lengths, they would be in the tail end of the distribution and hence contribute negligibly to the overall response. From $D \sim \xi_{y 0}$ and $\xi_{x 0} \approx \xi_{y 0} \approx 0.1 \xi_{z 0}$ (as estimated from the transverse tunneling probability in Sec. III), we have $\xi_{x 0}=\xi_{y 0} \cong 10^{2} \AA$ and $\xi_{z 0} \cong 10^{3} \AA$. It is interesting to note that (i) the estimated value of the longitudinal $\xi_{z 0}$ is on the smaller end of the typical coherence length, which can range from $10^{3}-10^{4} \AA$, and (ii) for $D \sim \xi_{y 0}$, the critical field $H_{c c} \sim\left(\lambda_{z 0} / D\right) H_{c}$ is of the same order as the upper critical field $H_{c 2} \sim \Phi_{o} / \xi_{y 0} \xi_{z 0} \sim\left(\lambda_{z 0} / \xi_{y 0}\right) H_{c}$, approaching the depairing field in a confined geometry of width $D \sim \xi_{y 0}{ }^{6,20}$

To compare simulation results with experimental data for energy gap and diamagnetic susceptibility, we need to estimate the dimensionless energy scale $\varepsilon_{0}=a_{o}^{2} T_{c}^{o 2} \delta_{0} / 2 b_{o} k_{B} T_{c}^{o}$, which is the zero-temperature condensation energy measured by $k_{B} T_{c}^{o}$ in the characteristic volume $\delta_{0}=\xi_{x 0} \xi_{y 0} \xi_{z 0}$. Physically, $\varepsilon_{o}$ measures the number of Cooper pairs in the volume $\delta_{0}$, given by $10^{-4} n \xi_{x 0} \xi_{y 0} \xi_{z 0} \sim 10$ according to the estimates above. ${ }^{21}$ According to the results in Sec. VI, this indicates that the phase-ordering transition temperature $T_{c}$ is very close to $T_{c}^{o}$, i.e., $T_{c} / T_{c}^{o} \cong 1$.

Based on the estimations made above, we have carried out numerical computation for comparison with experimental data, using $\varepsilon_{o} \sim 10$ and $D \sim \xi_{y 0}$ for the sample width. In Sec. VI the order parameters $\eta$ and $\gamma$ have been investigated as intrinsic properties for systems of sufficiently large size. The only control parameter there is the dimensionless energy scale $\varepsilon_{o}$. Here we focus on the finite-size effect on these properties. Figure 4 shows the gap parameter $\sqrt{\gamma}$ as a function of temperature, evaluated for different system sizes. The overall agreement with the experimental data is fairly satis-

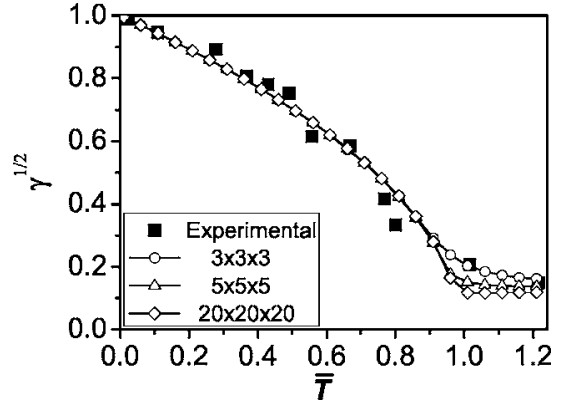

FIG. 4. The gap parameter $\sqrt{\gamma}$ as a function of temperature, evaluated for different system sizes, with $\varepsilon_{0}=10$.

factory. We want to point out that such an agreement can also be produced by using the 1D GL model. ${ }^{4}$ Here the weak dependence on dimensionality (and sample size) comes from the fact that the gap parameter measures the magnitude of local condensation, which is only weakly affected by the inter-wire coupling.

Figure 5 shows $|\langle\bar{m}\rangle| / \bar{B}$ as a function of temperature, evaluated at different values of the applied field $\bar{B}$ for a small system. The suppression of superconductivity can be seen from the decrease of $|\langle\bar{m}\rangle| / \bar{B}$ with increasing field strength, as observed experimentally. ${ }^{4}$ In particular, the diamagnetic response is almost completely suppressed when $\bar{B} \rightarrow 1$. Note that $\bar{B}$ (along the $x$ direction) is in units of $\Phi_{o} / \xi_{y 0} \xi_{z 0}$, which is of the same order as $H_{c c}$ and $H_{c 2}$ discussed above. If $\xi_{z 0} \cong 1000 \AA$ and $\xi_{y 0} \cong 100 \AA$, then $\bar{B}=1$ implies an applied field of $\sim 2$ Tesla. This is in rough accord with the experimentally observed value of 1.5 to 5 Tesla as the quenching field. In comparing the experimental data with the numerical results in Fig. 5, we note that in Ref. 4, the diamagnetic susceptibility has been normalized against 0.015 , the absolute value of the susceptibility measured at $T=1.6 \mathrm{~K}$ and $H=0.2$ Tesla. Considering that the volume used then in obtaining this value is the volume of the nanotubes only, which is an underestimate by a factor of $\sim 10$, and that the value of 0.015 is in units where $B=0$ corresponds to $\chi=-1$ rather than $\chi=-1 / 4 \pi$, we regard the data in Fig. 1 of Ref. 4 as the

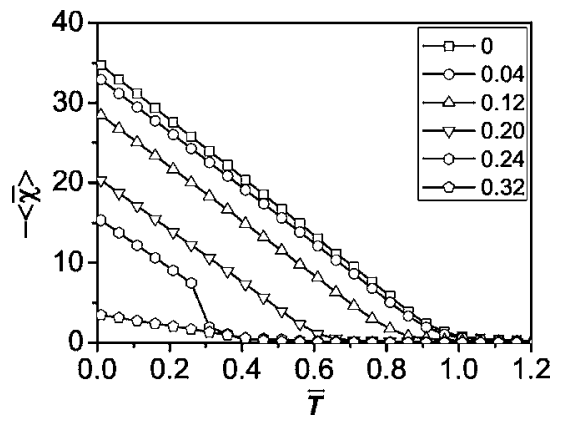

FIG. 5. $|\langle\bar{m}\rangle| / \bar{B}$ as a function of temperature, evaluated at different values of the applied field $\bar{B}$ for a small system of $\bar{L}_{x} \times \bar{L}_{y}$ $\times \bar{L}_{z}=3 \times 3 \times 3$, with $\varepsilon_{0}=10$. Here the vertical axis numbers are in the unit of $\hat{\chi}=\xi_{y 0}^{2} / 64 \pi^{3} \lambda_{z 0}^{2}$, estimated to be $10^{-7}$ in the case of nanotube-zeolite samples. 
(a)

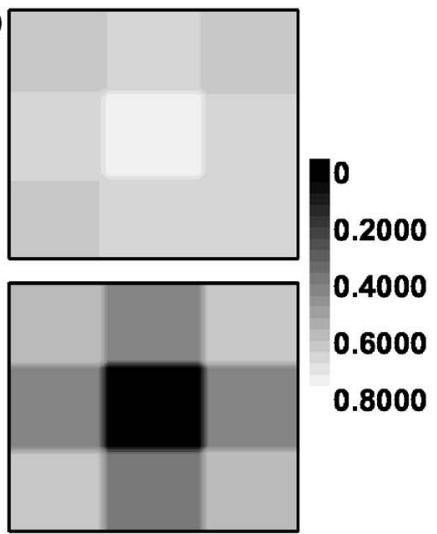

(b)

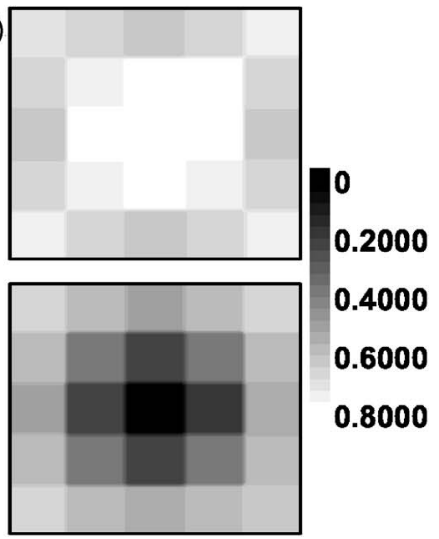

FIG. 6. The suppression of superconductivity in small systems where normal cores are induced by sufficiently high applied fields. (a) Cross-sectional distribution of $|\bar{\psi}|^{2}$ for a system of $\bar{L}_{x} \times \bar{L}_{y} \times \bar{L}_{z}$ $=3 \times 3 \times 3$ at $\bar{B}=0.2$ (upper panel) and 0.3 (lower panel). (b) Crosssectional distribution of $|\bar{\psi}|^{2}$ for a system of $\bar{L}_{x} \times \bar{L}_{y} \times \bar{L}_{z}=5 \times 5$ $\times 5$ at $\bar{B}=0.1$ (upper panel) and 0.2 (lower panel). The temperature is fixed at $\bar{T}=0.01$ and the parameter $\varepsilon_{0}$ fixed at 10 . Here the light color indicates a high value of $|\bar{\psi}|^{2}$, and the dark color indicates zero or vanishing $|\bar{\psi}|^{2}$. These pictures indicate the formation of vortices in the system, with the larger sample having a lower threshold magnetic field.

diamagnetic susceptibility normalized against the value of $10^{-4}$. The absolute value of the diamagnetic susceptibility can also be estimated to be on the order of $10^{-4} / 4 \pi$ using the penetration length $\lambda_{z 0} \approx 10^{4} \AA$ and the transverse size on the order of a few transverse coherence lengths, i.e., $D \sim \xi_{y 0}$ $\approx 100 \AA$. This is considered to be in rough accord with the experimental observation, though a difference of an order of magnitude is noted. Given that all the parameter values are presently only order-of-magnitude estimates, such discrepancy is probably not avoidable. In comparing Fig. 1 in Ref. 4 with Fig. 5 here, we note that those data actually correspond to the high-field regime since the applied field has to be large enough to make the diamagnetic moment $\langle m\rangle$ experimentally measurable. This is also seen by comparing the magnetic field values there with the field strength unit $\Phi_{o} / \xi_{y 0} \xi_{z 0} \approx 2$ Tesla here. However, most of the curves in Fig. 5 display a relatively linear variation with temperature, whereas experimentally the variation is much more nonlinear. A possible explanation for this discrepancy may come from the way in which the superconducting order parameter in a finite system is quenched with increasing magnetic field or temperature. In particular, the present model calculation uses a free energy functional in which a linear temperature dependence is assumed down to $T=0$ [through $\alpha=a_{o}\left(T-T_{c}^{o}\right)$ in $\left.F_{G L}[\psi]\right]$ although this linear dependence usually works only in the vicinity of $T_{c}^{o}$.

We plot in Fig. 6 the cross-sectional distribution of $|\bar{\psi}|^{2}$ for two small systems. It is seen that at weak applied field $|\bar{\psi}|^{2}$ is only slightly reduced at the boundaries. However, when the applied field is sufficiently strong, a normal core of $|\bar{\psi}|^{2} \approx 0$ is induced in the centre of the cross section (indicated by the dark color), resulting in a significant reduction of the diamagnetic response. The appearance of the normal core(s) represents a competition between the condensation energy and the kinetic energy: when the applied field is sufficiently large, the kinetic energy of a globally coherent system would be pushed so high that it would be energetically more favorable to lower the kinetic energy by sacrificing part of the condensation energy [via the normal core(s)]. Similar mechanism also works for ultrathin, doubly connected superconducting rings and cylinders. ${ }^{22,23}$ We want to point out that the normal cores numerically obtained here are vortices, around the center of which the phase of $\psi$ changes by an integral multiple of $2 \pi$, i.e., $\oint d \varphi=2 n \pi$, where $n$ is an integer close to $\Phi / \Phi_{o}$, with $\Phi$ being the magnetic flux penetrating the normal core. We have $n=1$ for the normal core shown in Fig. 6(a) and $n=2$ for that in Fig. 6(b). The sudden appearance of the normal core(s) is also reflected in the jump of the diamagnetic moment $\langle m\rangle$ (see the curve for $\bar{B}=0.24$ in Fig. $5)$. In a real sample that contains many disconnected superconducting domains with different geometric shapes, the jumps associated with the field-induced normal cores must be smeared by the averaging over differently shaped domains, thus leading to another potential reason for the nonlinear temperature dependence that was observed experimentally.

\section{DISCUSSION}

In this paper the anisotropic GL free energy functional has been used to model the system of coupled superconducting wires, with the intention of clarifying the superconducting behavior in $4 \AA$ nanotube-zeolite composites. ${ }^{4}$ Comparison with experimental data has been made by numerical evaluating of the Meissner effect for finite samples. Implications of our study are that (i) the sample quality needs to be improved before bulk superconducting behavior can be observed, (ii) the transverse coupling is reasonably strong between the carbon nanotubes, so that the observed superconducting behavior is that of an anisotropic superconductor with the zeolite matrix playing a very important role of regulating the intertube separations, (iii) consistent estimates of the material parameters can be obtained for the nanotube-zeolite composites, with $\lambda_{z 0} \approx 10^{4} \AA, \xi_{z 0} \approx 10^{3} \AA$, and $\xi_{x 0}=\xi_{y 0} \approx 10^{2} \AA$, and (iv) the resistance transition of a finite-sized superconducting region becomes observable only if it extends a few $\xi_{z 0}$ 's, e.g., 
$0.5 \mu \mathrm{m}$, along the alignment direction of the nanotubes. These results would serve as a guide for further experimental studies on the superconductivity in the $4 \AA$ nanotube-zeolite composites.

\section{ACKNOWLEDGMENTS}

We wish to acknowledge the support of Hong Kong RGC grants 602904, CA04/05.SC02, and 602805.
${ }^{1}$ L. X. Benedict, V. H. Crespi, S. G. Louie, and M. L. Cohen, Phys. Rev. B 52, 14935 (1995).

${ }^{2}$ D. J. Scalapino, M. Sears, and R. A. Ferrell, Phys. Rev. B 6, 3409 (1972).

${ }^{3}$ N. Wang, Z. K. Tang, G. D. Li, and J. S. Chen, Nature (London) 408, 50 (2000).

${ }^{4}$ Z. K. Tang, L. Zhang, N. Wang, X. X. Zhang, G. H. Wen, G. D. Li, J. N. Wang, C. T. Chan, and P. Sheng, Science 292, 2462 (2001).

${ }^{5}$ N. Metropolis, A. W. Rosenbluth, M. N. Rosenbluth, A. H. Teller, and E. Teller, J. Chem. Phys. 21, 1087 (1953).

${ }^{6}$ M. Kociak, A. Yu. Kasumov, S. Gueron, B. Reulet, I. I. Khodos, Yu. B. Gorbatov, V. T. Volkov, L. Vaccarini, and H. Bouchiat, Phys. Rev. Lett. 86, 2416 (2001).

${ }^{7}$ J. Gonzalez, Phys. Rev. B 67, 014528 (2003).

${ }^{8} \mathrm{M}$. Tinkham, Introduction to superconductivity, 2nd edition (McGraw Hill, New York, 1996).

${ }^{9}$ F. London, Phys. Rev. 74, 562 (1948).

${ }^{10}$ G. Blatter, V. B. Geshkenbein, and A. I. Larkin, Phys. Rev. Lett. 68, 875 (1992).

${ }^{11}$ Y. J. Feng and C. T. Chan (private communication).

${ }^{12}$ H. J. Liu and C. T. Chan, Phys. Rev. B 66, 115416 (2002). There are three bands crossing at the Fermi level for the $(5,0)$ carbon nanotube. The effective mass is calculated from the two bands which contribute to the dominant amount of density of states at the Fermi level.

${ }^{13}$ A. Schmid, Phys. Rev. 180, 527 (1969).

${ }^{14}$ D. E. McCumber and B. I. Halperin, Phys. Rev. B 1, 1054 (1970).

${ }^{15}$ C. Ebner and D. Stroud, Phys. Rev. B 39, 789 (1989).

${ }^{16}$ P. C. Hohenberg, Phys. Rev. 158, 383 (1967); N. D. Mermin and H. Wagner, Phys. Rev. Lett. 17, 1133 (1966).

${ }^{17}$ G. Deutscher, Y. Imry, and L. Gunther, Phys. Rev. B 10, 4598 (1974).

${ }^{18}$ C. Ebner and D. Stroud, Phys. Rev. B 25, 5711 (1982).

${ }^{19}$ W. J. Skocpol and M. Tinkham, Rep. Prog. Phys. 38, 1049 (1975).

${ }^{20}$ R. Meservey and P. M. Tedrow, Phys. Rep. 238, 173 (1994).

${ }^{21}$ For conventional superconductors, the typical value of $\varepsilon_{0}$ $\sim 10^{-4} n \xi_{0}^{3}$ ranges from $10^{3}$ to $10^{6}$, with the density of conducting electrons $n \sim 10^{22} / \mathrm{cm}^{3}$ and the typical coherence length $\xi_{0}$ $\sim 10^{3}-10^{4} \AA$.

${ }^{22}$ P. G. de Gennes, C. R. Seances Acad. Sci. III 292, 279 (1981).

${ }^{23}$ Y. Liu, Y. Zadorozhny, M. M. Rosario, B. Y. Rock, P. T. Carrigan, and H. Wang, Science 294, 2332 (2001). 